\title{
Discordant Bone Marrow Involvement in Diffuse Large B-Cell Lymphoma: Comparative Molecular Analysis Reveals a Heterogeneous Group of Disorders
}

\author{
Marcus Kremer, Martin Spitzer, Sonja Mandl-Weber, Katrin Stecker, \\ Burkhard Schmidt, Heinz Höfler, Leticia Quintanilla-Martínez, and Falko Fend \\ Institutes of Pathology (MK, MS, SM-W, KS, HH, FF) and Department of Internal Medicine III (BS), Technical University, \\ Munich, and GSF-National Research Center for Health and Environment (MK, LQ-M), Neuherberg, Germany
}

SUMMARY: Discordant bone marrow (BM) involvement in patients with a diagnosis of large-cell non-Hodgkin's lymphoma (NHL) is characterized by marrow infiltrates predominantly composed of small lymphoid cell, cytologically compatible with low-grade $\mathrm{NHL}$. Although this phenomenon is well described morphologically, molecular data concerning the relationship of the two lesions are lacking. The aim of the study was to investigate the clonal relationship of discordant lymphoma manifestations by using immunoglobulin heavy chain gene $(\mathrm{IgH})$, as well as bcl-2 rearrangements, as molecular markers. IgH rearrangements were amplified by PCR with consensus primers directed against framework regions 3 or 2 (FR3 and FR2), followed by automated fragment length analysis and sequencing in selected cases. Rearrangements of the bcl-2 gene were identified with primers against the major breakpoint region. Small BM infiltrates were isolated by laser capture microdissection. In addition, immunohistochemistry was performed on paraffin sections using antibodies against CD3, CD10, CD20, bcl-2, bcl-6, p53, and the Ki67 antigen. Paraffin-embedded tissues of 21 cases diagnosed as diffuse large B-cell lymphoma (DLBCL) with discordant BM involvement and no previous history of low-grade B-cell NHL were analyzed. After review of immunohistochemical stains, 5 cases were excluded either as concordant BM infiltrates by large-cell lymphoma with abundant reactive T-cells ( 2 cases) or as benign, reactive lymphoid infiltrates ( 3 cases), as confirmed by a polyclonal pattern in the IgH analysis. Of the remaining 16 cases, a common clonal origin was confirmed in 8 cases by the presence of an identical clonal lgH rearrangement or bcl-2 rearrangement. In 4 cases, identification of distinct lgH or bcl-2 rearrangements gave evidence for the presence of two clonally unrelated neoplasms. The remaining 4 cases were not evaluable for technical reasons. Morphological, phenotypical, and molecular findings were compatible with a lymphoma of germinal center origin in the majority of cases. However, in 4 cases, flow cytometric analysis of the BM infiltrates revealed a B-cell chronic lymphocytic leukemia phenotype. Two of these cases were clonally related to the DLBCL and thus represented Richter's transformation. In summary, discordant BM infiltrates in DLBCL represent a heterogeneous group of disorders, encompassing cases with a clonally related, clinically occult small-cell component, as well as cases with two clonally distinct, unrelated B-cell neoplasms presenting synchronously at different locations. (Lab Invest 2003, 83:107114).

S ignificant variations in the cytologic composition $S$ of neoplastic infiltrates in different biopsy sites is a well-recognized phenomenon in malignant lymphomas. Discordant morphology has been documented in 14 to $38 \%$ of patients undergoing multiple biopsies. Most frequently, however, discordance is documented as a bone marrow (BM) infiltrate predominantly composed of small cells, morphologically compatible with infiltration by low-grade non-Hodgkin's lymphoma (NHL), in patients with large-cell lymphoma in an extramedullary site (Bartl et al, 1988; Fisher et al, 1981, 1989; Hodges et al, 1994; Kluin et al, 1990; Robertson et al, 1991). The reasons for BM discor-

\section{DOI: 10.1097/01.LAB.0000050762.61660.27}

Received September 26, 2002.

Supported in part through a grant from the Wilhelm Sander-Stiftung, Germany (LQ-M and FF).

Address reprint requests to: Dr. Falko Fend, Institute of Pathology, Technical University Munich, Ismaninger Strasse 22, D-81675 Munich, Germany.E-mail:fend@lrz.tum.de dance are unclear, but proposed mechanisms include tumor evolution or transformation of a low-grade NHL, preferential recirculation of small lymphoid tumor cells, differences in the microenvironment, or the presence of two unrelated neoplasms (Fisher et al, 1989; Hodges et al, 1994; Kluin et al, 1990; Robertson et al, 1991). The clinical implications of discordant BM infiltrates are not well studied, but some reports have suggested that these patients have remission rates and survival similar to those without BM involvement (Fisher et al, 1989; Hodges et al, 1994; Robertson et al, 1991). On the other hand, there may be an increased risk for late relapse in patients who have apparently achieved complete remission after therapy (Robertson et al, 1991). Beyond the prognostic implications of BM discordance, it is still unclear whether predominantly small-cell infiltrates in the $\mathrm{BM}$ of patients with large-cell lymphoma really represent a manifestation of the same clonal process.

Although the phenomenon of discordant BM infiltrates is well described morphologically, molecular 
data are sparse, and comparative analyses to determine the clonal relationship of the two lesions are lacking (Crisan and Mattson, 1995; Nakano et al, 1992). Because discordant BM infiltration is predominantly recognized in patients with diffuse large B-cell lymphoma (DLBCL), analysis of IgH rearrangements allows us to compare the "molecular signatures" of the neoplastic cells. The detection of clonal PCR products of identical size in the BM and lymph node (LN) can be considered as evidence for an expansion of the same clone in different sites, regardless of differences in morphology. An additional suitable marker of clonal relatedness is the $t(14 ; 18)(q 32 ; q 21)$ translocation, which occurs in up to $30 \%$ of DLBCL, and can indicate a follicular origin of the lymphoma (Weiss et al, 1987).

The aim of this study was to elucidate the clonal relationship of BM infiltrates and the primary DLBCL in patients with discordant disease by comparative analysis of $\mathrm{lgH}$ - and $\mathrm{Bcl}-2$ gene rearrangements.

\section{Results}

\section{Patient Characteristics}

The clinical data are summarized in detail in Table 1. Of the 21 patients, 10 were male and 11 female with a median of 60 years (range, 30-78 years). Patients were treated with combined radiation and chemotherapy modalities in 4 cases, chemotherapy and peripheral stem cell transplantation in 3 cases, and chemotherapy alone in 14 cases. Nine patients died with disease. Twelve patients are alive, including 3 recent cases with insufficient follow-up time. Five patients have persistence or recurrence of low-grade NHL, and 4 patients have no evidence of disease. The mean follow-up period was 25.2 months (range, 2-120 months).

\section{Histologic Findings}

The investigated DLBCL showed sheets of large centroblasts or immunoblasts with large nuclei and prominent nucleoli and basophilic cytoplasm and occasional small lymphocytes. None of the cases showed evidence of a follicular growth pattern or a low-grade component (Fig. 1A).

The lymphoid infiltrates in the investigated BM biopsies consisted of small- to medium-sized lymphocytes with dense nuclear chromatin and scant cytoplasm embedded in an increased reticulin network. Centroblasts were either absent or represented only a small minority of the infiltrate. Two main patterns of infiltration were observed. Peritrabecular infiltrates typical for follicular lymphoma (FL) were present in 14

Table 1. Clinical Features

\begin{tabular}{|c|c|c|c|c|c|}
\hline Case & Age & Sex & Treatment & $\begin{array}{l}\text { Follow-up } \\
\text { outcome }\end{array}$ & Months \\
\hline \multicolumn{6}{|c|}{ Group 1: clonally identical $(n=8)$} \\
\hline 1 & 55 & M & CT & DwD & 60 \\
\hline 2 & 60 & $\mathrm{~F}$ & CT & DwD & 12 \\
\hline 3 & 78 & M & CT & $A w D^{a}$ & 17 \\
\hline 4 & 36 & M & CT & $A w D^{a}$ & 19 \\
\hline 5 & 67 & $\mathrm{~F}$ & CT & ANED & 12 \\
\hline 6 & 60 & $\mathrm{~F}$ & $\mathrm{CT}+\mathrm{RT}$ & recent & 9 \\
\hline 7 & 30 & $\mathrm{~F}$ & $\mathrm{CT}+\mathrm{PST}$ & recent & 5 \\
\hline 8 & 53 & $\mathrm{~F}$ & $\mathrm{CT}+\mathrm{RT}$ & $\mathrm{DwD}$ & 9 \\
\hline \multicolumn{6}{|c|}{ Group 2: clonally unrelated $(n=4)$} \\
\hline 9 & 66 & M & CT & $A w D^{a}$ & 16 \\
\hline 10 & 30 & M & CT+PST & $\mathrm{AwD}^{b}$ & 58 \\
\hline 11 & 48 & M & $\mathrm{CT}+\mathrm{RT}$ & ANED & 48 \\
\hline 12 & 45 & M & $\mathrm{CT}+\mathrm{PST}$ & DwD & 6 \\
\hline \multicolumn{6}{|c|}{ Group 3: technically not evaluable $(n=4)$} \\
\hline 13 & 73 & $\mathrm{~F}$ & CT & DwD & 5 \\
\hline 14 & 73 & $\mathrm{~F}$ & CT & DwD & 10 \\
\hline 15 & 73 & M & CT & DwD & 7 \\
\hline 16 & 65 & $\mathrm{~F}$ & CT & $A w D^{a}$ & 12 \\
\hline \multicolumn{6}{|c|}{ Cases excluded (concordant BM infiltrates [17, 18]; reactive BM infiltrates [19-21]) } \\
\hline 17 & 67 & $\mathrm{M}$ & CT & DwD & 4 \\
\hline 18 & 78 & M & CT & DwD & 2 \\
\hline 19 & 65 & $\mathrm{~F}$ & CT & ANED & 120 \\
\hline 20 & 68 & $\mathrm{~F}$ & $\mathrm{CT}+\mathrm{RT}$ & ANED & 90 \\
\hline 21 & 77 & $\mathrm{~F}$ & CT & recent & 9 \\
\hline
\end{tabular}

$\mathrm{CT}=$ chemotherapy; RT = radiotherapy; PST = peripheral stem-cell transplantation; DwD = died with disease; AwD = alive with disease; ANED = alive with no evidence of disease; recent $=$ recently diagnosed case.

${ }^{a}$ Persistence of low-grade non-Hodgkin's lymphoma.

${ }^{b}$ Recurrence of low-grade non-Hodgkin's lymphoma. 

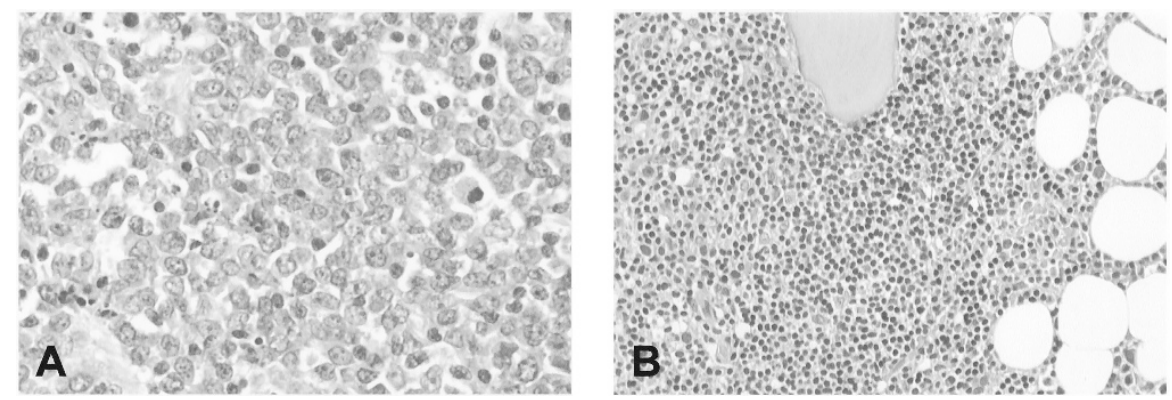

Figure 1.

Case 2, diffuse large B-cell lymphoma (DLBCL) with discordant bone marrow (BM) infiltration. A, Lymph node (LN) biopsy specimen with diffuse large B-cell lymphoma (hematoxylin and eosin $\times 400$ ). B, Discordant BM involvement with peritrabecular infiltrate of small lymphoid cells with rare blasts (hematoxylin and eosin $\times 200)$.

cases (Fig. 1B). Seven cases showed centrally located, dense infiltrates with a more homogeneous lymphocyte population and indistinct borders. The 3 control cases with morphologically reactive lymphoid infiltrates showed well circumscribed, centrally located nodules of small lymphocytes with intermingled plasma cells, without reticulin fibrosis.

\section{Immunophenotypic Findings}

All DLBCL cases expressed CD20 and were negative for CD3. The neoplastic cells of the DLBCL showed $\mathrm{Bcl}-2$ protein expression in 18 of 21 cases (85\%), Bcl-6 in 13 of 21 cases (61\%), and CD10 also in 13 of 21 cases (61\%). P53 protein expression in greater than $20 \%$ of the tumor cells was identified in 9 cases (42\%).

The BM infiltrates showed a preponderance of CD20-positive small lymphocytes in 16 of 21 cases (76\%). The remaining 5 cases were reclassified. Three of them showed a predominance of CD3-positive T-lymphocytes with few CD20-positive small B-lymphocytes within the infiltrates. On the basis of the central location and the phenotype of the infiltrates, these 3 cases were reclassified as reactive infiltrates (cases 19-21). This reclassification was supported by a polyclonal rearrangement pattern in the molecular analysis (see paragraph below). The other two biopsies, which had previously been classified morphologically as discordant infiltrates, revealed a minority of large CD20 positive blasts without small B-cells in a background of abundant CD3 positive small lymphocytes. Accordingly, they were reclassified as concordant BM infiltrates of the DLBCL, with a major reactive T-cell population (cases 17 and 18). These 5 cases were excluded from further analysis.

Data of flow cytometric immunophenotyping performed in parallel was available in 18 cases (Table 2 ). Involvement by low-grade B-NHL was evident in only 9 cases. Four cases were classified as B-CLL (cases $1,3,9$, and 16) on basis of the coexpression of CD5 and CD23; all showed centrally located marrow infiltrates in histology. The remaining 5 diagnostic cases were classified as compatible with an involvement by low-grade B-NHL, not otherwise specified. Case 17, reclassified as $B M$ involvement by $D L B C L$, showed a clonal population of large B-cells by flow cytometry, compatible with this diagnosis. Eight cases showed no evidence for lymphoma, including 2 cases reclassified as reactive in which flow immunophenotyping had been performed (data not shown).

\section{Molecular Findings}

The remaining 16 patients after exclusion of the 5 reclassified cases could be divided into 3 groups according to the results of the $\mathrm{IgH}$ - and bcl-2 rearrangement analysis (Table 2 ).

\section{Group 1: Clonally Related Cases}

An identically sized clonal rearrangement of the $\mathrm{lgH}$ chain genes (with either FR3- or FR2 primers) or the bcl-2 gene in both locations was demonstrated in 8 of 12 evaluable cases (67\%) by Genescan analysis. Four cases showed an identical FR3, 2 cases an identical FR2 rearrangement, and 4 cases showed identical bcl-2 rearrangements in both the investigated $L N$ and the BM, respectively (Fig. 2). In 2 cases (5 and 8) the bcl-2 gene rearrangement was the only comparable clonal marker identified. Two of the cases with a diagnosis of B-CLL in the BM by flow cytometry belonged to this group. Both were negative for a bcl-2 rearrangement.

\section{Group 2: Clonally Unrelated Cases}

In 4 of 12 evaluable cases (33\%), the analysis of LN and $\mathrm{BM}$ revealed distinct clonal amplification products. Three cases reproducibly showed FR3 products of different size and direct sequencing confirmed unrelated clonal products (Fig. 3). One of the B-CLL cases diagnosed by flow cytometry of the BM belonged to this group (case 9). Case 12 revealed bcl-2 amplification products of different length in $\mathrm{LN}$ and $\mathrm{BM}$, but failed to show clonality with FR3 and FR2 primers.

\section{Group 3: Technically Unevaluable Cases}

Four cases were considered unevaluable for technical reasons. In one case, both samples showed a polyclonal pattern with both $\mathrm{lgH}$ primer sets and were 
Table 2. Immunophenotypic and Molecular Results

\begin{tabular}{|c|c|c|c|c|c|c|c|c|c|c|}
\hline \multirow[b]{2}{*}{ Case } & \multirow[b]{2}{*}{ Site } & \multicolumn{3}{|c|}{ Molecular results } & \multicolumn{5}{|c|}{ Immunohistochemistry } & \multirow[b]{2}{*}{ Flow cytometry (BM) } \\
\hline & & FR3 & FR2 & Bcl-2 & CD20 & CD10 & $\mathrm{Bcl}-2$ & $\mathrm{Bcl}-6$ & p53 & \\
\hline \multicolumn{11}{|c|}{ Group 1: clonally identical $(n=8)$} \\
\hline \multirow[t]{2}{*}{1} & LN & 102 & n.d. & neg & pos & pos & neg & neg & neg & \\
\hline & BM & 102 & n.d. & n.d. & pos• & & & & & B-CLL (CD19+, CD5+, CD23+, kappa) \\
\hline \multirow[t]{2}{*}{2} & LN & 88 & n.d. & neg & pos & neg & +++ & neg & neg & \\
\hline & $\mathrm{BM}$ & 88 & n.d. & n.d. & pos• & & & & & no evidence of lymphoma \\
\hline \multirow[t]{2}{*}{3} & EN & poly & 245 & neg & pos & pos & + & + & + & \\
\hline & BM & n.e. & 245 & n.d. & pos• & & & & & B-CLL (CD19+, CD5+, CD23+, kappa) \\
\hline \multirow[t]{2}{*}{4} & LN & 82 & n.d. & pos $^{*}$ & pos & pos & +++ & ++ & neg & \\
\hline & BM & 82 & n.d. & $\operatorname{pos}^{*}$ & pos. & & & & & no evidence of lymphoma \\
\hline \multirow[t]{2}{*}{5} & EN & poly & pos & $\operatorname{pos}^{*}$ & pos & pos & +++ & + & neg & \\
\hline & BM & n.e. & n.e. & $\operatorname{pos}^{*}$ & pos. & & & & & low-grade B-NHL (CD19+, CD20+, CD10+) \\
\hline \multirow[t]{2}{*}{6} & EN & poly & 237 & neg & pos & pos & +++ & + & + & \\
\hline & BM & poly & 237 & neg & pos. & & & & & $\begin{array}{c}\text { low-grade B-NHL (CD19+, CD20+, CD22+, } \\
\text { CD10+) }\end{array}$ \\
\hline \multirow[t]{2}{*}{7} & EN & 78 & n.d. & $\operatorname{pos}^{*}$ & pos & pos & +++ & + & neg & \\
\hline & BM & 78 & n.d. & pos $^{*}$ & pos• & & & & & $\begin{array}{l}\text { low-grade B-NHL (CD19+, CD20+, CD22+, } \\
\text { CD10+, kappa) }\end{array}$ \\
\hline \multirow[t]{2}{*}{8} & EN & poly & n.d. & pos $^{*}$ & pos & neg & ++ & + & +++ & \\
\hline & $\mathrm{BM}$ & poly & n.d. & $\operatorname{pos}^{*}$ & pos• & & & & & no evidence of lymphoma \\
\hline \multicolumn{11}{|c|}{ Group 2: clonally unrelated $(n=4)$} \\
\hline \multirow[t]{2}{*}{9} & LN & $103 \infty$ & n.d. & neg & pos & pos & + & neg & neg & \\
\hline & BM & $115 \infty$ & n.d. & neg & pos• & & & & & B-CLL (CD19+, CD20+, CD5+, CD23+, lambda) \\
\hline \multirow[t]{2}{*}{10} & EN & $97 \infty$ & n.d. & neg & pos & pos & + & + & ++ & \\
\hline & BM & $85 \infty$ & n.d. & n.d. & pos• & & & & & low-grade B-NHL (CD19+, CD20+, CD10+, kappa) \\
\hline \multirow[t]{2}{*}{11} & EN & $105 \infty$ & n.d. & neg & pos & neg & +++ & ++ & neg & \\
\hline & BM & $94 \infty$ & n.d. & pos & pos• & & & & & low-grade B-NHL (CD19+, CD20+, CD10+, kappa) \\
\hline \multirow[t]{2}{*}{12} & LN & poly & pos & pos\# & pos & pos & +++ & + & ++ & \\
\hline & $\mathrm{BM}$ & poly & n.e. & pos\# & pos• & & & & & no evidence of lymphoma \\
\hline \multicolumn{11}{|c|}{ Group 3: technically not evaluable $(n=4)$} \\
\hline \multirow[t]{2}{*}{13} & LN & 94 & pos & neg & pos & pos & + & ++ & neg & \\
\hline & $\mathrm{BM}$ & poly & n.e. & n.d. & pos• & & & & & not done \\
\hline \multirow[t]{2}{*}{14} & EN & 100 & n.d. & pos & pos & pos & +++ & neg & neg & \\
\hline & BM & n.e. & n.e. & n.e. & pos• & & & & & no evidence of lymphoma \\
\hline \multirow[t]{2}{*}{15} & LN & n.e. & n.e. & n.e. & pos & neg & + & neg & + & \\
\hline & BM & 111 & n.d. & neg & pos• & & & & & no evidence of lymphoma \\
\hline \multirow[t]{2}{*}{16} & LN & poly & poly & neg & pos & pos & +++ & neg & + & \\
\hline & BM & poly & poly & neg & pos• & & & & & B-CLL (CD19+, CD20+, CD5+, CD23+, kappa+) \\
\hline
\end{tabular}

$\mathrm{LN}=$ Iymph node; EN = extranodal; BM = bone marrow; poly = polyclonal rearrangement pattern; n.d. $=$ not done; n.e. $=$ not evaluable; pos ${ }^{\star}=$ bcl-2 amplification products of the same size; pos\# = bcl-2 amplification products of different sizes; $\infty$ sequenced amplification products; pos. = in BM biopsies, only CD20 and CD3 was performed.

negative for a bcl-2 rearrangement, thus lacking a comparable clonal marker. In the remaining three cases, DNA from one of the two biopsy sites failed to amplify.

\section{Controls}

The four FL with concordant BM involvement showed $\mathrm{lgH}$ gene rearrangements of identical size in the $L N$ as well as in the BM with FR3 primers. The analysis of the three cases of DLBCL with morphologically reactive lymphoid infiltrates in the BM revealed a monoclonal amplification product in all three lymphoma specimens, whereas the microdissected reactive infiltrates in the corresponding BM biopsies showed a polyclonal $\mathrm{lgH}$ gene rearrangement pattern (data not shown). Similarly, the three cases reclassified as reactive BM infiltrate were polyclonal by IgH PCR.

\section{Discussion}

Our study demonstrates that discordant BM involvement in DLBCL represents a heterogeneous group of disorders. The comparative molecular analysis of the two disease manifestations provided evidence for the expansion of a single B-cell clone with divergent morphologies in the majority of evaluable cases. However, in a third of the informative cases, discordant BM infiltrates in patients with DLBCL seem to represent a second, clonally unrelated low-grade B-NHL.

Histologically discordant BM infiltrates primarily composed of small cells is a well-recognized phenom- 


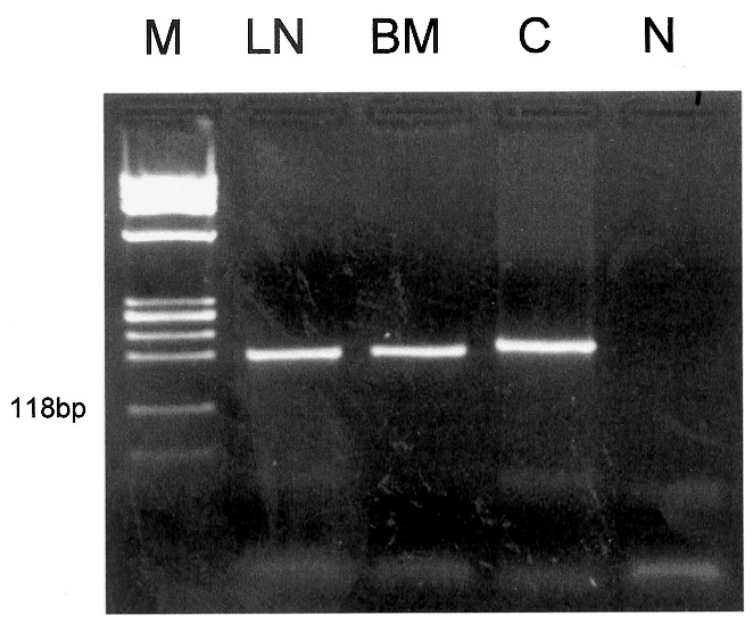

Figure 2.

Case 5, clonally related discordant BM infiltrate. Detection of the bcl-2 rearrangements in LN and corresponding BM. PCR amplification with bcl-2 primer pairs. The LN and BM lanes show amplified bands of equal size. $C=$ Follicular lymphoma with bcl-2 rearrangement as positive control. $N=$ Negative control.

enon in patients with DLBCL. It has been found in 14 to $38 \%$ of patients with DLBCL and BM involvement (Bartl et al, 1988; Fisher et al, 1981, 1989; Hodges et al, 1994; Juneja et al, 1990; Kluin et al, 1990; Mead et al, 1983; Robertson et al, 1991). However, "discordant" or "divergent" BM infiltration has not been well defined. Many authors include both predominantly small-cell as well as mixed BM infiltrates in patients with large-cell lymphoma under this term. If, in addition, cases of FL with different cytologic grades at the two biopsy sites are included, the reported incidence of discordance reaches 50 to $60 \%$ of cases (Fisher et al, 1989; Hodges et al, 1994; Jagannath et al, 1985).

Even when strict criteria are applied, such as in our study, discordant BM involvement occurs at a high frequency in patients with DLBCL. Of the 42 patients with DLBCL and BM involvement evaluated for this study, only $15(36 \%)$ had a concordant BM involvement by large-cell lymphoma, including the 2 reclas- sified cases. Sixteen (38\%) of the 42 cases met our criteria for discordance. The remaining 11 cases were excluded for a variety of reasons, including a previous history of low-grade lymphoma and misinterpretations of benign infiltrates as involvement by lymphoma. These findings underline the importance of careful morphological evaluation, including immunohistochemical stainings, before a diagnosis of discordant $\mathrm{BM}$ infiltration is made. Another relevant observation was the high (38\%) false-negative rate of flow cytometric investigation in our series, which documents the necessity for trephine BM biopsy for the staging of patients with lymphoma. This is especially true for FL, which usually shows peritrabecular infiltrates and reticulin fibrosis, as in all our cases, negative by flow cytometry (Hanson et al, 1999).

Although discordant BM infiltration is generally interpreted as involvement by the same clonal process, phenotypical or molecular evidence to support this hypothesis is remarkably sparse. Only a handful of studies have addressed this problem, and none of them investigated the clonal relationship in a systematic way. A single study investigated $\mathrm{lgH}$ gene and T-cell receptor gene rearrangements in discordant BM infiltrates by Southern blotting and found clonal populations in most cases classified as lymphoma infiltrates. However, the primary lymphoma was not available for comparative analysis in that series (Crisan and Mattson, 1995).

We therefore devised a strategy to investigate this frequent clinical problem on the molecular level in a retrospective study. Comparative analysis of rearranged IgH genes in both biopsy sites allows elucidation of the clonal relationship of the two populations and can be applied to paraffin-embedded tissues. To ensure optimal sensitivity and specificity, we used laser capture microdissection for the isolation of small BM infiltrates and combined it with automated fragment-length analysis of PCR products. This approach has proven successful for the identification of small clonal populations in BM biopsies in a previous study (Kremer et al, 2000). In addition to lgH gene
A

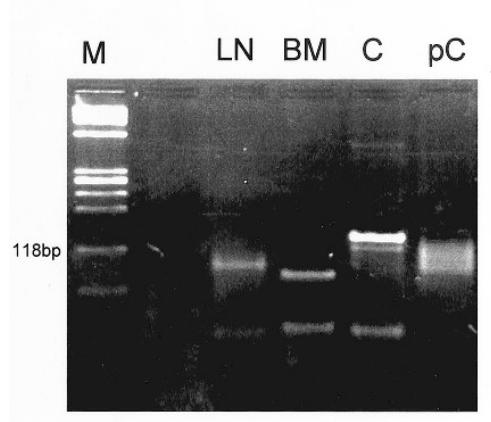

B

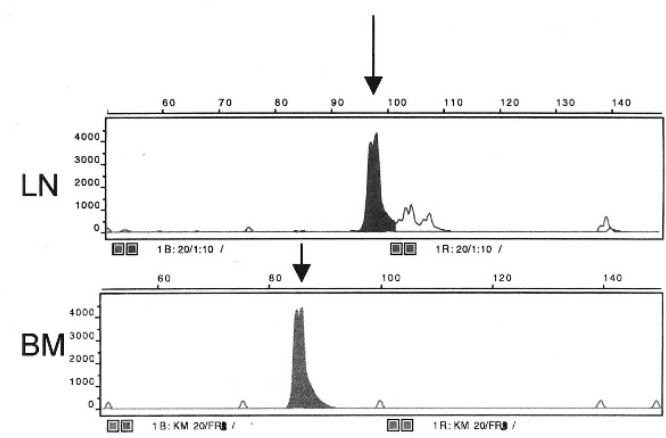

Figure 3.

Case 9, clonally unrelated discordant BM infiltrate. A, Detection of clonal IgH-gene rearrangements of different size in nodal DLBCL (LN) and corresponding bone marrow biopsy (BM). PCR amplification with FR3 primers. $M=$ Molecular weight marker. $C=$ Monoclonal control. $p C=L N$ with reactive changes as polyclonal control. B, Computer-assisted fragment length analysis (Genescan) confirmed the different sizes of the IgH-gene rearrangements. LN, 97 base pairs; BM, 85 base pairs (arrows). 
analysis, we performed PCR for bcl-2 rearrangements because they can be found in up to $30 \%$ of DLBCL cases and can serve as an additional clonal marker. Furthermore, many cases of discordant BM infiltration are thought to be related to $F L$, and the presence of bcl-2 rearrangements can serve to substantiate this assumption.

Using the approach outlined above, we were able to demonstrate that two thirds of evaluable discordant $\mathrm{NHL}$ infiltrates represent the same clone, with identical $\mathrm{IgH}$ or bcl-2 gene rearrangements as the large-cell lymphoma. In our series, $75 \%$ of these cases are related to $\mathrm{FL}$, as based on the morphology of the BM infiltrates, the immunophenotype of the DLBCL, and the high frequency of bcl-2 rearrangements. Therefore, these cases could be regarded as transformation of clinically occult FL. Because primary FL transforms to DLBCL in 30 to $60 \%$ of cases during the course of the disease, this finding is not surprising. In secondary DLBCL arising from FL, a common clonal origin can be demonstrated in most of the cases (de Jong et al, 1989; Matolcsy, 1999; Matolcsy et al, 1999; Zelenetz et al, 1991). Although we have not sequenced the $\lg \mathrm{H}$ amplification products of the eight cases in this group, identical base-pair sizes by automated fragment length analysis strongly support a common clonal origin of the two lesions. In addition, identical bcl-2 rearrangements, as seen in four of the cases, are consistent with this hypothesis. Bcl-2 rearrangements are constant markers of the neoplastic clone in FL and are preserved throughout the course of the disease (Raffeld et al, 1987). The expression of p53 protein in $37 \%$ of the high-grade component in this group would also fit well with secondary high-grade transformation because alterations of p53 are frequent in transformed FL (Sander et al, 1993).

In two of the cases with common clonal origin (cases 1 and 3), the low-grade component in the BM showed a B-CLL phenotype with coexpression of CD5+ and CD23+ by flow cytometric immunophenotyping. In contrast to the other cases, they showed centrally located nodules rather than peritrabecular infiltrates composed of monomorphic small lymphocytes, which is consistent with a diagnosis of B-CLL. Nevertheless, DLBCL with discordant BM infiltration as primary manifestation of Richter's syndrome is an unusual finding given the low incidence of high-grade transformation in B-CLL.

Interestingly, in a third of the evaluable cases, we obtained evidence for two clonally distinct neoplasms. In three cases, we reproducibly obtained FR3 amplicons of different sizes. Sequence analysis confirmed the presence of two unrelated clones, rather than internal deletions or insertions, as a cause for the different product lengths. It is unlikely that the two sequences are because of differential amplification of biallelic rearrangements derived from the same neoplastic cell because the two products were reproducibly associated with the different tumor manifestations (Fend et al, 1999). In case 12, the presence of two different bcl-2 rearrangements confirmed a distinct clonal origin despite the absence of reproducible clonal $\mathrm{IgH}$ rearrangements in the BM. Of note is that DLBCL arising in the setting of a low-grade B-NHL does not necessarily represent transformation of the low-grade tumor. For instance, Matolcsy et al (1994) demonstrated distinct clonal origins in two of six cases of B-CLL with Richter's syndrome, which is consistent with our finding of separate clonal origins in one of the cases with B-CLL.

The clinical follow-up data available for our patients are insufficient to allow firm conclusions about the prognostic significance of discordant BM infiltrates. Nevertheless, it is probably justified to state that the presence of discordant disease, irrespective of the clonal relatedness, should not be equated with disseminated (stage IV) large-cell lymphoma for therapeutic purposes. This conservative approach is also justified by previous reports (Fisher et al, 1989; Hodges et al, 1994; Robertson et al, 1991). The persistence or recurrence of the low-grade component in three of our patients also supports the notion that these patients are at an increased risk for late relapse and probably require a follow-up strategy more suited for indolent lymphoma. In addition, the frequent occurrence of clonally unrelated B-cell proliferations in this setting indicates that a more detailed evaluation of discordant infiltrates may help to avoid misinterpretations of BM findings, especially if flow cytometry or molecular methods are used for staging and follow-up.

Although comparative molecular studies of discordant BM infiltrates have not been published to date, a recent study of discordant BM infiltrates using flow cytometry also found evidence for two distinct neoplasms in a third of the cases (Wright et al, 2002). Similar results were obtained in a larger series of $\mathrm{NHL}$ patients in whom any two different sites with lymphoma infiltrates were studied by flow immunophenotyping (Onciu et al, 2002).

In summary, discordant BM infiltration in DLBCL represents a heterogeneous group of disorders. Although both tumor components are clonally related in the majority of cases, and the DLBCL might be regarded as transformation of a clinically inapparent low grade NHL, there is evidence of a distinct clonal B-cell proliferation in a significant minority of cases.

\section{Materials and Methods}

\section{Tissue Samples}

A retrospective search of the computer-based registry of the Department of Pathology of the Technical University of Munich was conducted to identify patients with DLBCL and BM involvement of any type, for whom paraffin blocks of both biopsy sites were available. Clinical information was obtained from the patients' medical records. All slides were reviewed and reclassified by two pathologists (FF and MK) according to the World Health Organization classification (Jaffe et al, 2001). Forty-two patients with DLBCL and $\mathrm{BM}$ involvement at the time of diagnosis were identified. After pathologic review of routinely stained sec- 
tions and evaluation of the patients' medical records, 21 patients were excluded from the study for the following reasons: concordant BM infiltration by large cell infiltrates (13 cases, 30\%), a history of low-grade $\mathrm{NHL}$ (5 cases), insufficient material for analysis (1 case), morphologically reactive lymphoid infiltrates in the BM (1 case), and unclassifiable primary lymphoma (1 case). Twenty-one patients with morphologically discordant BM infiltrates remained for analysis.

LNs and BM biopsies of four cases with low-grade $\mathrm{FL}$ and $\mathrm{BM}$ infiltration as well as three patients with DLBCL and morphologically reactive lymphoid BM infiltrates were analyzed as controls.

The primary diagnosis of DLBCL was made on LN biopsies $(n=10)$ and extranodal tumor infiltrates $(n=$ 11) thoroughly characterized by paraffin section immunostaining. BM samples were either formaldehyde(4\%) $(\mathrm{pH} 7.4)$ or formaldehyde (1\%)/glutaraldehyde $(0.4 \%)$-fixed $(\mathrm{pH} 7.4)$ for at least 24 hours and decalcified with buffered sodium-ethylenediaminetetraacetic acid ( $\mathrm{pH}$ 7.0) for 48 hours. One micrometer thick sections were cut, stained with hematoxylin and eosin, Giemsa, Gomori's reticulin stain, and Naphthol AS-D chloroacetate esterase.

\section{Immunohistochemistry}

All primary lymphoma biopsies were stained for CD20 (L26, Dako, Copenhagen, Denmark), CD3 (polyclonal, Dako), CD10 (Novocastra, Newcastle, United Kingdom), Bcl-2 (Dako), Bcl-6 (Novocastra), and p53 (Dako). Additional stains for CD5, kappa, and lambda light chains and other markers were performed when considered necessary. The proliferation rate was assessed by staining for the Ki67 antigen (MIB1, Dako). Because the fixation in a $1 \%$ formaldehyde/glutaraldehyde fixation used for the majority of the investigated BM biopsies results in reduced immunoreactivity, the trephine biopsies were stained only for CD20 and CD3.

Immunohistochemistry was performed on an automated immunostainer (Ventana Medical systems, Tucson, Arizona) according to the manufacturer's protocols, with minor modifications. After deparaffinization and rehydration, the slides were placed in a microwave pressure cooker in $0.01 \mathrm{~mol} / \mathrm{L}$ citrate buffer ( $\mathrm{pH}$ 6.0) containing $0.1 \%$ Tween 20 and heated in a microwave oven at maximum power $(800 \mathrm{~W})$ for 30 minutes. The sections were immediately cooled in Tris-buffered saline and washed in 3\% goat serum for 20 minutes. Incubations with the primary antibodies were performed overnight at room temperature. The rest of the procedure was completed on the Ventana immunostainer. Positive controls for all investigated antibodies were used to confirm the adequacy of the staining. Lymphomas were scored as p53-positive if greater or equal to $20 \%$ of the tumor cells had nuclear staining.

\section{Laser Capture Microdissection}

Laser capture microdissection was performed on all BM biopsies in which the lymphoid infiltrates made up only a minor part of the total biopsy volume. Serial sections were stained for CD20 and microdissected using a Pix Cell II laser capture microdissection device with an infrared diode laser (Acturus Engineering, Santa Clara, California) as described elsewhere (Fend et al, 1999; Kremer et al, 2000). At least five lymphoid nodules were pooled per biopsy. In some cases in which the lymphoid infiltrates disappeared in the serial sections, original Giemsa or Naphtol AS-D chloroacetate esterase-stained sections were microdissected after removal of the coverslip. Whole serial sections were used for DNA extraction, when more extensive $\mathrm{BM}$ infiltrates were present.

\section{Molecular Analysis}

Genomic DNA was extracted from the LN and BM biopsies and used to assay for clonal rearrangements of the $\mathrm{IgH}$ and $\mathrm{Bcl}-2$ genes by PCR using published protocols (Segal et al, 1994; Stetler-Stevenson et al, 1988). The tissues were digested with proteinase $K$ overnight at $56^{\circ} \mathrm{C}$, and the crude extract was used for PCR after heat denaturation. Primers directed against consensus sequences of the framework regions 3 and 2 (FR3 and FR2) of the variable regions of the lg heavy chain genes and consensus sequences of the joining regions were used. Fluorescence-labeled downstream primers were employed for automated fragment length analysis. FR3 analysis was performed as a single-step PCR with 40 cycles of amplification (Segal et al, 1994). A seminested PCR protocol was employed with the FR2 primers performing 35 and 25 cycles of amplification, respectively (Fend et al, 1999; Ramasamy et al, 1992). Two microliters of the crude extract served as template for the first round of amplification, and $4 \mu \mathrm{L}$ of the diluted external PCR product was used for the second round using the same temperature profile. PCR analysis of the bcl-2 translocation was performed with primers directed at the major break region as previously described (Fend et al, 1999; Stetler-Stevenson et al, 1988). All PCR reactions were run at least in duplicate to ensure reproducibility. Adequate positive and negative controls were included in every run. PCR reaction products were electrophoresed in a 3\% Metaphor gel (Biozym Diagnostik, Oldendorf, Germany). In addition, all fluorescence-labeled $\mathrm{lgH}$ amplification products were analyzed with an automated fragment length analysis system using a high-resolution polyacrylamide gel for size determination (Genescan; Perkin Elmer, Weiterstadt, Germany) (Kremer et al, 2000; Worley et al, 1997).

In cases that showed IgH PCR products of different sizes in the two locations, clonal amplification products were extracted from agarose gel slices using the QIAquick gel extraction Kit (Qiagen, Hilden, Germany) and directly sequenced using the BigDye Terminator cycle sequencing Kit (Perkin Elmer, Weiterstetten, Germany) and an ABI Prism 377 automated sequencer. 


\section{Acknowledgment}

The authors are grateful to Jacqueline Müller, Sandra Rath, Elenore Samson, and Nadine Kink for their excellent technical assistance.

\section{References}

Bartl R, Hansmann ML, Frisch B, and Burkhardt R (1988). Comparative histology of malignant lymphomas in lymph node and bone marrow. Brit J Haematol 69:229-237.

Crisan D and Mattson JC (1995). Discordant morphologic features in bone marrow involvement by malignant lymphomas: Use of gene rearrangement pattern for diagnosis. Am J Hematol 49:299-309.

de Jong D, Voetdijk BMH, van Ommen GJB, and Kluin PM (1989). Alterations in immunoglobulin genes reveal the origin and evolution of monotypic and bitypic B cell lymphomas. Am J Pathol 134:1233-1242.

Fend F, Quintanilla-Martínez L, Kumar S, Beaty MW, Blum L, Sorbara L, Jaffe ES, and Raffeld M (1999). Composite low grade B-cell lymphomas with two immunophentotypically distinct cell populations are true biclonal lymphomas. Am J Pathol 154:1857-1866.

Fisher DE, Jacobson JO, Ault KA, and Harris NL (1989). Diffuse large cell lymphoma with discordant bone marrow histology. Cancer 64:1879-1887.

Fisher RI, Jones RB, DeVita VT, Simon RM, Garvin AJ, Berard CW, and Young RC (1981). Natural history of malignant lymphomas with divergent histologies at staging evaluation. Cancer 47:2022-2025.

Hanson CA, Kurtin PJ, Katzmann JA, Hoyer JD, Li C-Y, Hodnefield JM, Meyers $\mathrm{CH}$, Habermann TM, and Witzig TE (1999). Immunophenotypic analysis of peripheral blood and bone marrow in the staging of B-Cell malignant lymphoma. Blood 94:3889-3896.

Hodges GF, Lenhardt TM, and Cotelingam JD (1994). Bone marrow involvement in large-cell lymphoma. Am J Clin Pathol 101:305-311.

Jaffe ES, Harris NL, Stein H, and Vardiman JW (2001). World Health Organization classification of tumours. Pathology and genetics of tumours of haematopoietic and lymphoid tissues. Lyon, France: IARC Press.

Jagannath S, Velasquez WS, Tucker SL, Manning JT, McLaughlin P, and Fuller LM (1985). Stage IV diffuse largecell lymphoma: A long-term analysis. J Clin Oncol 3:39-47.

Juneja SK, Wolf MM, and Cooper IA (1990). Value of bilateral bone marrow biopsy specimens in non-Hodgkin's lymphoma. J Clin Pathol 43:630-632.

Kluin PM, van Krieken JH, Kleiverda K, and Kluin-Nelemans HC (1990). Discordant morphologic characteristics of B-cell lymphomas in bone marrow and lymph node biopsies. Am J Clin Pathol 94:59-66.

Kremer M, Cabras AD, Fend F, Schulz S, Schwarz K, Hoefler $\mathrm{H}$, and Werner $\mathrm{M}$ (2000). PCR analysis of IgH-gene rearrangements in small lymphoid infiltrates microdissected from sections of paraffin-embedded bone marrow specimens. Hum Pathol 31:847-853.

Matolcsy A (1999). High-grade transformation of low-grade non-Hodgkin's lymphomas: mechanisms of tumor progression. Leuk Lymphoma 34:251-259.
Matolcsy A, Inghirami G, and Knowles DM (1994). Molecular genetic demonstration of the diverse evolution of Richter's syndrome (chronic lymphocytic leukemia and subsequent large cell lymphoma). Blood 83:1363-1372.

Matolcsy A, Schattner EJ, Knowles DM, and Casali P (1999). Clonal evolution of B-cells in transformation from low- to high-grade lymphoma. Eur J Immunol 29:1253-1264.

Mead GM, Kushlan P, O'Neil M, Burke JS, and Rosenberg SA (1983). Clinical aspects of non-Hodgkin's lymphomas presenting with discordant histologic subtypes. Cancer 52: 1496-1501.

Nakano M, Kawanishi Y, Kuge S, Kuriyama Y, Kuwabara S, Yaguchi M, and Toyama K (1992). Clinical and prognostic significance of monoclonal small cells in the peripheral blood and bone marrow of various B-cell lymphomas. Blood 79: 3253-3260.

Onciu M, Berrak SG, Medeiros LJ, Katz RL, and Huh YO (2002). Discrepancies in the immunophenotype of lymphoma cells in samples obtained simultaneously from different anatomic sites. Am J Clin Pathol 117:644-650.

Raffeld M, Wright JJ, Lipford E, Cossman J, Longo DL, Bakhshi A, and Korsmeyer SJ (1987). Clonal evolution of $t(14 ; 18)$ follicular lymphomas demonstrated by immunoglobulin genes and the 18q21 major breakpoint region. Cancer Res 47:2537-2542.

Ramasamy I, Brisco M, and Morley A (1992). Improved PCR method for detecting monoclonal immunoglobulin heavy chain rearrangement in B cell neoplasms. J Clin Pathol 45:770-775.

Robertson LE, Redman JR, Butler JJ, Osborne BM, Velasquez WS, McLaughlin P, Swan F, Rodriguez MA, Hagemeister FB, Fuller LM, and Cabanillas F (1991). Discordant bone marrow involvement in diffuse large-cell lymphoma: $A$ distinct-pathologic entity associated with a continuous risk of relapse. J Clin Oncol 9:236-242.

Sander CA, Yano T, Clark HM, Harris C, Longo DL, Jaffe ES, and Raffeld M (1993). P53 mutation is associated with progression in follicular lymphomas. Blood 82:1994-2004.

Segal GH, Jorgensen T, Scott M, and Braylan RC (1994). Optimal primer selection for clonality assessment by polymerase chain reaction analysis. II. Follicular lymphomas. Hum Pathol 25:1276-1282.

Stetler-Stevenson M, Raffeld M, Cohen P, and Cossman J (1988). Detection of occult follicular lymphoma by specific DNA amplification. Blood 72:1822-1825.

Weiss LM, Warnke RA, Sklar J, and Cleary ML (1987). Molecular analysis of the $t(14 ; 18)$ chromosomal translocation in malignant lymphomas. N Engl J Med 317:1185-1189.

Worley J, Lee S, Ma MS, Eisenberg A, Chen HY, and Mansfield $E$ (1997). Fluorescence imaging in human identity testing. Biotechniques 23:148-153.

Wright BF, Asplund SL, McKenna RW, and Kroft SH (2002). Large B-cell lymphoma with discordant marrow involvement: An immunophenotypic analysis of 11 cases (Abstract). Mod Pathol 15:270A

Zelenetz AD, Chen TT, and Levy R (1991). Histologic transformation of follicular lymphoma to diffuse lymphoma represents tumor progression by a single malignant cell. J Exp Med 173:197-207. 